OPEN ACCESS

Edited by:

Dimitrios Petrou Bogdanos, University of Thessaly, Greece

Reviewed by:

Matthew Stoll,

University of Alabama at Birmingham, United States

Deepika Sharma

University of Chicago, United States

${ }^{*}$ Correspondence:

Tim B. van der Houwen

t.vanderhouwen@erasmusmc.nl

Jan A. M. van Laar

j.vanlaar@erasmusmc.nl

Specialty section:

This article was submitted to Autoimmune and Autoinflammatory

Disorders,

a section of the journal

Frontiers in Immunology

Received: 30 December 2019

Accepted: 13 May 2020

Published: 12 June 2020

Citation:

van der Houwen TB, van Laar JAM,

Kappen $J H$, van Hagen $P M$,

de Zoete MR, van Muijlwijk GH,

Berbers R-M, Fluit AC, Rogers M,

Groot J, Hazelbag CM, Consolandi C, Severgnini M, Peano C, D'Elios MM,

Emmi G and Leavis HL (2020)

Behçet's Disease Under Microbiotic Surveillance? A Combined Analysis of

Two Cohorts of Behçet's Disease

Patients. Front. Immunol. 11:1192.

doi: 10.3389/fimmu.2020.01192

\section{Behçet's Disease Under Microbiotic Surveillance? A Combined Analysis of Two Cohorts of Behçet's Disease Patients}

Tim B. van der Houwen ${ }^{1 *}$, Jan A. M. van Laar ${ }^{1 *}$, Jasper H. Kappen ${ }^{2,3}$, Petrus M. van Hagen ${ }^{1}$, Marcel R. de Zoete ${ }^{4}$, Guus H. van Muijlwijk ${ }^{4}$, Roos-Marijn Berbers ${ }^{5}$, Ad C. Fluit ${ }^{4}$, Malbert Rogers ${ }^{4}$, James Groot ${ }^{4}$, C. Marijn Hazelbag ${ }^{6}$, Clarissa Consolandi ${ }^{7}$, Marco Severgnini ${ }^{7}$, Clelia Peano ${ }^{8,9}$, Mario M. D'Elios $^{10}$, Giacomo Emmi ${ }^{10}$ and Helen L. Leavis ${ }^{5}$

${ }^{1}$ Section Clinical Immunology, Departments of Internal Medicine and Immunology, Erasmus University Medical Center Rotterdam, Rotterdam, Netherlands, ${ }^{2}$ Allergy and Clinical Immunology, Immunomodulation and Tolerance Group, Inflammation Repair and Development, Imperial College, National Heart and Lung Institute, London, United Kingdom, ${ }^{3}$ Department of Pulmonology, STZ Centre of Excellence for Asthma and COPD, Franciscus Group, Rotterdam, United Kingdom, ${ }^{4}$ Medical Microbiology, University Medical Center Utrecht, Utrecht, Netherlands, ${ }^{5}$ Department of Rheumatology and Clinical Immunology, University Medical Center Utrecht, Utrecht, Netherlands, ${ }^{6}$ Department of Epidemiology, Julius Center for Health Sciences and Primary Care, University Medical Center Utrecht, Utrecht, Netherlands, ${ }^{7}$ National Research Council, Institute of Biomedical Technologies, Segrate, Italy, ${ }^{8}$ National Research Council, Institute of Genetic and Biomedical Research, UoS Milan, Milan, Italy, ${ }^{9}$ Genomic Unit, Humanitas Clinical and Research Center, Milan, Italy, ${ }^{10}$ Department of Experimental and Clinical Medicine, University of Firenze, Firenze, Italy

Background: In Behçet's disease (BD), an auto-inflammatory vasculitis, an unbalanced gut microbiota can contribute to pro-inflammatory reactions. In separate studies, distinct pro- and anti-inflammatory bacteria associated with BD have been identified.

Methods: To establish disease-associated determinants, we performed gut microbiome profiling in BD patients from the Netherlands $(n=19)$ and Italy $(n=13)$, matched healthy controls $(\mathrm{HC})$ from the Netherlands $(n=17)$ and Italy $(n=15)$ and oral microbiome profiling in Dutch BD patients $(n=18)$ and $\mathrm{HC}(n=15)$ by $16 \mathrm{~S}$ rRNA gene sequencing. In addition, we used fecal IgA-SEQ analysis to identify specific IgA coated bacterial taxa in Dutch BD patients $(n=13)$ and $\mathrm{HC}(n=8)$.

Results: In BD stool samples alpha-diversity was conserved, whereas beta-diversity analysis showed no clustering based on disease, but a significant segregation by country of origin. Yet, a significant decrease of unclassified Barnesiellaceae and Lachnospira genera was associated with BD patients compared to HC. Subdivided by country, the Italian cohort displays a significant decrease of unclassified Barnesiellaceae and Lachnospira genera, in the Dutch cohort this decrease is only a trend. Increased IgA-coating of Bifidobacterium spp., Dorea spp. and Ruminococcus bromii species was found in stool from BD patients. Moreover, oral Dutch BD microbiome displayed increased abundance of Spirochaetaceae and Dethiosulfovibrionaceae families.

Conclusions: BD patients show decreased fecal abundance of Barnesiellaceae and Lachnospira and increased oral abundance of Spirochaetaceae and Dethiosulfovibrionaceae. In addition, increased fecal IgA coating of Bifidobacterium, 
Ruminococcus bromii and Dorea may reflect retention of anti-inflammatory species and neutralization of pathosymbionts in $\mathrm{BD}$, respectively. Additional studies are warranted to relate intestinal microbes with the significance of ethnicity, diet, medication and response with distinct pro- and inflammatory pathways in BD patients.

Keywords: Behçet's disease, microbiota, intestinal, oral, IgA-SEQ

\section{INTRODUCTION}

Behçet's disease (BD) is an auto-inflammatory vasculitis, characterized by mucosal ulcerations, skin lesions and uveitis (1). Immunosuppressives are indicated in organ or vision threatening cases (2). BD seems to be driven by an excessive T-cell reaction that might be triggered by an infectious antigen in a genetically susceptible host (3). This is emphasized in the association with HLA-B51 and non-HLA genetic associations like IL10, IL23R, and ERAP1, which suggests a genetic susceptibility similar to spondyloarthritis (4). Increased salivary colonization with Streptococcus mutans has been demonstrated in BD patients and both skin and serum testing with streptococcal antigens induce inflammatory reactions (5-8). Furthermore, antibiotics added to regular treatment has yielded a significant improvement of symptoms in BD patients (9). Subsequently, dysbiosis of the Gut Microbiome (GM) might trigger or exacerbate inflammatory processes in $\mathrm{BD}$ patients.

Dysbiotic GM, showing, for example a reduced intestinal microbial diversity, has been observed in BD patients before. Specifically, significant depletion of Roseburia and Subdoligranulum species leading to decreased butyrate production has been suggested to contribute to inflammation (10). Furthermore, a significant increase of Bifidobacterium and Eggerthella genera and reduction of Megamonas and Prevotella genera have been reported in a Japanese BD cohort compared with matched healthy controls (HC) (11). Rothia dentocariosa, a known abundant inhabitant of the oral cavity of BD patients, was found decreased in active ulcerations sites. Moreover, oral Neisseria and Veillonella numbers were decreased in both active and inactive BD (12). These studies demonstrate that different pro- and anti-inflammatory oral and gut bacteria, among different $\mathrm{BD}$ populations, may contribute as potential disease making determinants. Furthermore, identification of specific immunoglobulin A-(IgA-) coated bacteria may aid in identification of pro- or anti-inflammatory commensals by IgA-SEQ analysis (13). For instance, in Crohn's disease (CD) and spondylarthritis, a selective enrichment in IgA-coated Escherichia coli was found, which induced a mucosal and systemic Th17 response when administered in mice (14). In this observational study on a Dutch cohort, we aimed to extend insights into GM and Oral Microbiome (OM) in BD. We compared this cohort to an Italian one, and performed IgA-SEQ analysis in fecal samples of Dutch BD patients.

Abbreviations: BD, Behçet's disease; HC, Healthy controls; GM, Gut microbiome; $\mathrm{CD}$, Crohn's disease; Ig, Immunoglobulin; IT, Italy; NL, the Netherlands; OM, Oral microbiome; PCoA, Principle Coordinate Analysis; PCA, Principle Component Analysis; SCFA, Short-chain fatty acid.

\section{METHODS}

\section{Ethics Statement}

Ethic approval for this study was received from the Medical Ethical Committee of the Erasmus MC (MEC-2012-220). Written informed consent was obtained from all patients and controls according to the Declaration of Helsinki.

\section{Study Design}

In this observational study, patients were selected at the outpatient clinic of the Erasmus MC in Rotterdam, the Netherlands or the outpatient clinic of the Florence Behçet Center, Florence, Italy. All patients were diagnosed according to the International Study Group of BD criteria (15). Only patients aged 18 years or older were included. Use of proton pump inhibitors or any antibiotics in the 3 months preceding the study was used as exclusion criteria. Healthy controls included family members and non-family members, matched for ethnic background. Inclusion and exclusion criteria for the Italian cohort are the same as those described in Consolandi et al. (10) and are overlapping in the use of antibiotics as exclusion criteria.

\section{Sample Collection and DNA Extraction}

In the Dutch cohort, samples were taken from non-ulcerated tissue around the inferior labial frenulum using oral swabs [Copan swab $482 \mathrm{CE}$ with Amies medium (Murrieta, CA, USA)] and stored at $-80^{\circ} \mathrm{C}$. Stool samples [Sterilin UK specimen container, Thermo-Fisher (Waltham, Massachusetts, USA)] were gathered at the outpatient clinic or at patients' homes. Samples were transferred on dry ice and stored at $-20^{\circ} \mathrm{C}$ within $24 \mathrm{~h}$, for a maximum of 14 days, when samples were transported to $-80^{\circ} \mathrm{C}$. DNA was extracted from stool, for both the Dutch and the Italian cohort, with QIAamp DNA Stool Mini Kit (QIAGEN, Valencia, CA, USA) and from oral swabs with AGOWA mag Mini DNA Isolation Kit (AGOWA, Berlin, Germany) as previously described $(16,17)$.

\section{S rRNA Gene Sequencing}

The extracted DNA, for both oral and fecal samples, was sequenced following the protocol described before (18). Briefly, a 469 bp amplicon encompassing the V3 and V4 hypervariable regions of the 16S rRNA gene was amplified and sequenced using the Illumina MiSeq Reagent Kit v3 $2 \times 300$ bp (600-cycle) or the Illumina MiSeq Reagent Kit v2 $2 \times 250$ bp (500-cycle) on a MiSeq Illumina sequencer according to Fadrosh et al. (19). To monitor potential contamination during DNA extraction, amplification and sequencing protocol negative- and buffer controls were used. 


\section{Bioinformatic Data Analysis}

In order to have identical read lengths for all samples, reads from the $2 \times 300 \mathrm{bp}$ run were trimmed down to $250 \mathrm{bp}$ to match the other dataset. All reads were pre-processed as described (18) and merged using FLASH (version 1.2.11) (20) with options "-t 1" and "-M 167." Subsequently, merged reads were de-multiplexed using the "split_libraries_fastq.py" script and further analyzed using the QIIME microbial community analysis pipeline (version 1.9.1) (21). Quality filtering was also performed, using the split_library_fastq.py script in qiime, with the following non-default settings: q20 (truncating reads with an average PHRED quality score of 20 or less), max_bad_run_length 50 (trimming reads after 50 following bases with an average PHRED quality score of 20 or less), barcode_type24, max_barcode_errors 3. After removal of the barcodes, heterogeneity spacers, and primer sequences, about 6.2 million sequences were left, with a mean length of $409 \mathrm{bp}$ (median length of $404 \mathrm{bp}$ ). Sequences were assigned to operational taxonomic units (OTUs) at $97 \%$ similarity using QIIME's open-reference OTU picking workflow ("pick_open_reference_otus.py") and the USEARCH package (version 6.1.544) (22), in addition to detection and removal of chimeric sequences (which is performed by UCHIIME as part of the USEARCH package). The OTU sequences were aligned to the Greengenes 16S rRNA gene database (13.8 release), followed by removal of OTUs represented by $<0.005 \%$ of the total number of sequences, which was 5.4 million. The average number of sequences per samples was 73,900 (median 80,100). The generated OTU table was used for assessing alpha- and betadiversity using QIIME's core_diversity_analyses.py workflow with a rarefaction depth of 10,800 sequences, chosen based on the lowest amount of sequences while maintaining the most amount of samples as possible.

\section{IgA-SEQ Analysis}

IgA-SEQ analysis was performed as described before (13). Briefly, bacteria in fecal samples were stained with PE-conjugated AntiHuman IgA (Miltenyi Biotec clone IS-8E10, Cologne, Germany) prior to fluorescence-activated cell sorting flow cytometric analysis and sorting of IgA positive bacteria. After isolation of bacterial DNA from 200,000 sorted bacteria (both IgA positive and IgA negative), $16 \mathrm{~S}$ rRNA sequencing of the V4 region was performed on an Illumina miSeq $(2 \times 250)$ using barcoded primers (23). IgA Coating Index scores were calculated by dividing the relative abundance of a species in the $\operatorname{IgA}+$ fraction by the relative abundance of that species in the IgA- fraction.

\section{Statistical Data Analysis}

For Principal Component Analysis (PCA), R 3.5.0 within RStudio 1.1.463 (RStudio Team, Boston, MA, USA) (24) was employed, using zCompositions, clr transformation and ggplot $\mathrm{R}$ packages $(25,26)$. Principal Coordinate Analysis (PCoA) with Bray-Curtis distance was used to calculate beta-diversity of the samples, with permutational multivariate analysis of variance (PERMANOVA) to test for statistical significance. Shannon index was used to calculate alpha-diversity with Wilcoxon Rank to test for statistical significance.
Microbial diversity and statistical analyses for IgA-SEQ were performed with QIIME (v. 1.8.0), the Vegan package for R, and LEfSe $(21,27)$.

Microbiota changes between $\mathrm{BD}$ patients, $\mathrm{HC}$ and the different cohorts (patients subdivided by country of origin or HLAB51 status) were investigated using ANCOM (28) in R 3.3.3 (24). For statistical testing, we used false discovery rates (FDRs) correction for multiple comparisons and a FDR-adjusted $p$-value $<0.05$ was considered significant (29).

\section{RESULTS}

\section{Patients and Data Characteristics}

Patients and HC were included in a Dutch and an Italian cohort. The Dutch cohort consisted of 19 patients [mean age: 50 years (range: 34-65)] and 17 age-, gender- and ethnicity-matched HC; the Italian cohort of 14 patients [mean age: 45 (range: $30-71$ )] and 15 age-, gender- and ethnicity matched HC. From all patients and HC, stool samples were taken. Oral samples were provided by 18 Dutch patients and 15 Dutch HC.

Although both patient cohorts showed comparable disease manifestations, notable differences in medication usage were present. In the Italian cohort, corticosteroids were significantly $(p<0.01)$ more often prescribed (Table 1).

\section{Characterization of GM in BD Patients and $\mathrm{HC}$}

Using a cut-off value defined at 10,800 reads, 64 samples were selected (1 sample excluded because of too few sequence reads) for subsequent analyses. To study microbial alpha-diversity, Shannon index was calculated, showing no significant differences between 32 patients [Netherlands (NL) $n=19$, Italy (IT) $n=13$ ] and $32 \mathrm{HC}$ (NL $n=17$, IT $n=15)$. The Italian cohort displayed a significant lower alpha-diversity $(\mathrm{p}=0.006)$, compared to the

TABLE 1 | Patient characteristics.

\begin{tabular}{|c|c|c|c|c|}
\hline & \multicolumn{2}{|c|}{ Dutch cohort } & \multicolumn{2}{|c|}{ Italian cohort } \\
\hline & Patients & Controls & Patients & Controls \\
\hline & $n=19$ & $n=17$ & $n=13$ & $n=15$ \\
\hline Age $^{\star}$ & $50(34-65)$ & 45 & $45(30-71)$ & 44 \\
\hline Female $^{\star \star}$ & 53 & 47 & 54 & 53 \\
\hline \multicolumn{5}{|c|}{ Behçet's disease symptoms ${ }^{\star \star}$} \\
\hline - oral ulceration & 100 & - & 100 & - \\
\hline - genital ulceration & 84 & - & 69 & - \\
\hline - skin lesions & 79 & - & 85 & - \\
\hline - uveitis & 37 & - & 31 & - \\
\hline - HLAB51 positive & 47 & - & 54 & - \\
\hline \multicolumn{5}{|l|}{ Medication use ${ }^{\star \star}$} \\
\hline - colchicine & 74 & - & 62 & - \\
\hline - biologicals & 16 & - & 38 & - \\
\hline - corticosteroids & 5 & - & 77 & - \\
\hline
\end{tabular}

*Years (mean, range). ${ }^{\star \star}$ Expressed as \%. 
Dutch cohort. Moreover, in Italian patients, a trend toward a decreased alpha-diversity was observed compared to their HC (Figures 1A,B).

PCoA demonstrated a similar beta-diversity, with no distinct clustering of patients or HC. A significant $(p<0.001)$ segregation between patients based on country of origin was observed, as also shown in the PCoA plot displaying only healthy controls from both countries (Figure 1B).

ANCOM analysis was used to identify differences in GM between $\mathrm{BD}$ patients and $\mathrm{HC}$ at taxonomical levels of family and genus.

A depletion of the relative abundance of the family Barnesiellaceae in $\mathrm{BD}$ patients was found compared to $\mathrm{HC}$. In separate analysis of the Italian and Dutch cohorts, only the Italian $\mathrm{BD}$ patients displayed a significant decrease in
Barnesiellaceae. In the Dutch cohort the decrease was observed as a trend. In addition, an increase in the relative abundance of the family of Lactobacillaceae was shown for the Italian cohort compared to healthy controls. This was not observed in the Dutch cohort (Figure 2). Analysis at genus level in the combined cohort revealed a significant decrease of relative abundance in unclassified Barnesiellaceae and in Lachnospira in BD patients compared with HC (Figure 2). This decrease in Lachnospira was also significant in separate analysis of Italian BD patients, where in Dutch patients this decrease was only observed as a trend.

Further analysis, subdividing patients into cohorts by clinical parameters, showed a distinct microbiome in HLAB51-positive patients. In these patients, ANCOM analysis displayed a significant decrease in relative abundance of the family of Barnesiellaceae and two unclassified families of the Clostridiales

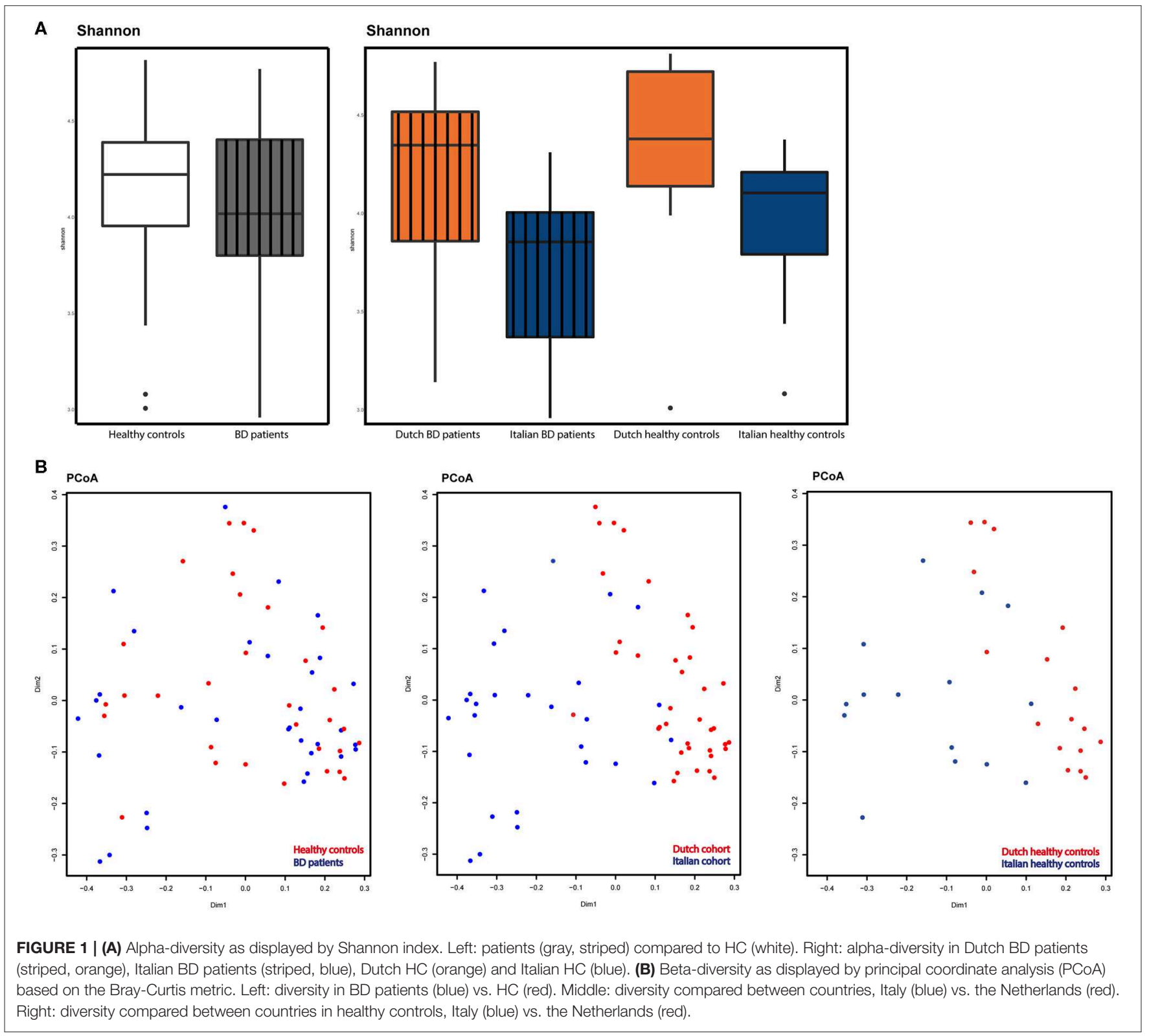




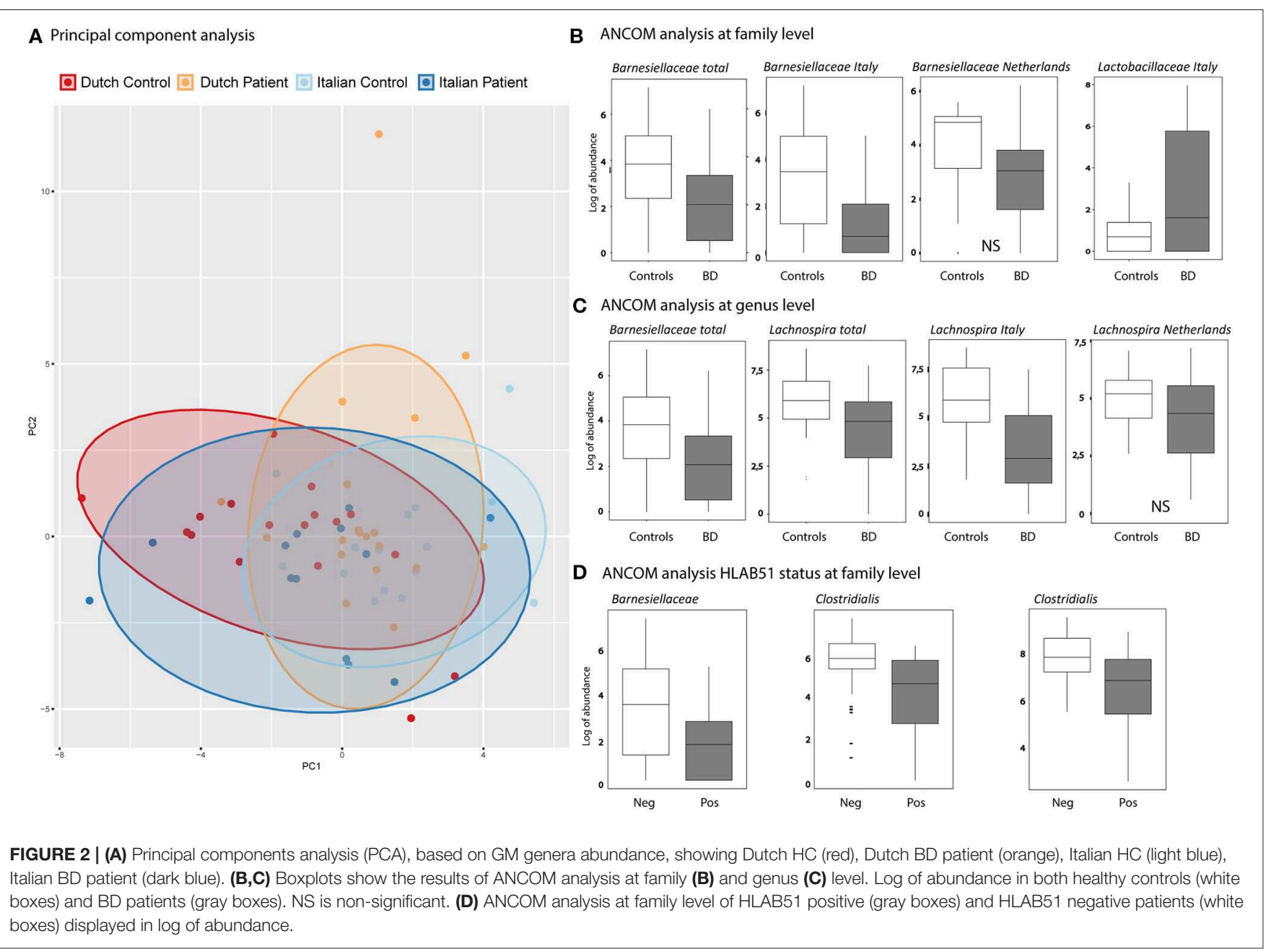

order (Figure 2). Other subgrouping of patients based on disease manifestations was not contributing.

On fecal samples from 13 patients and $8 \mathrm{HC}$ of the Dutch cohort, IgA-SEQ analysis was performed and showed distinct IgA coating of taxa in $\mathrm{BD}$ and $\mathrm{HC}$, not found by traditional $16 \mathrm{~S}$ rDNA sequencing. In Figures $\mathbf{3 A}, \mathbf{B}$ the differences in the heatmaps of traditional 16s rDNA sequencing and IgA-Coating Index are displayed. Three species showed increased IgA coating in $\mathrm{BD}$ patients as compared to HC: Bifidobacterium spp, Dorea spp and Ruminococcus bromii spp. (Figure 3C). In contrast, HC showed an increase in IgA inducing Erysipelotrichaceae spp. and RF39 spp. as compared to BD patients. To clarify, the overall abundance of this species is displayed in Table 2, no significant differences in abundance of this species were observed.

\section{Oral Microbiome Profiling in Dutch BD Patients}

Oral samples of 18 patients were compared with oral samples of 15 matched and HC, all from the Dutch cohort. Alpha-diversity was not significantly different between BD patients and HC. PCA revealed similar $\beta$-diversity, with no distinct clustering of patients from HC (Figure 4). By ANCOM analysis, oral samples from
TABLE 2 | IgA-SEQ analysis, relative abundance of species with significant differences in IgA coating.

\begin{tabular}{lll}
\hline Taxonomic units & $\begin{array}{c}\text { Behçet's disease } \\
\text { Average relative abundance } \\
\text { (standard deviation) }\end{array}$ \\
\hline Bifidobacterium spp. & $0.0867(0.11)$ & $0.0387(0.03)$ \\
Rominucoccus bromii spp. & $0.0088(0.01)$ & $0.0065(0.01)$ \\
UC Dorea spp. & $0.0002(0.00)$ & $0.0004(0.00)$ \\
RF39 spp. & $0.0001(0.00)$ & $0.0018(0.00)$ \\
Erysipelotrichaceae spp. & $0.0074(0.00)$ & $0.0056(0.01)$
\end{tabular}

patients with $\mathrm{BD}$ were enriched in the family of Spirochaetaceae and Dethiosulfovibrionaceae (Figure 4). Analysis at genus level revealed a significant increase in the relative abundance of both Treponema and TG5 in patients with BD (Figure 4).

\section{DISCUSSION}

In combined Dutch and Italian cohorts, alpha and beta diversity were not different from $\mathrm{HC}$, yet patients with $\mathrm{BD}$ demonstrated 
A

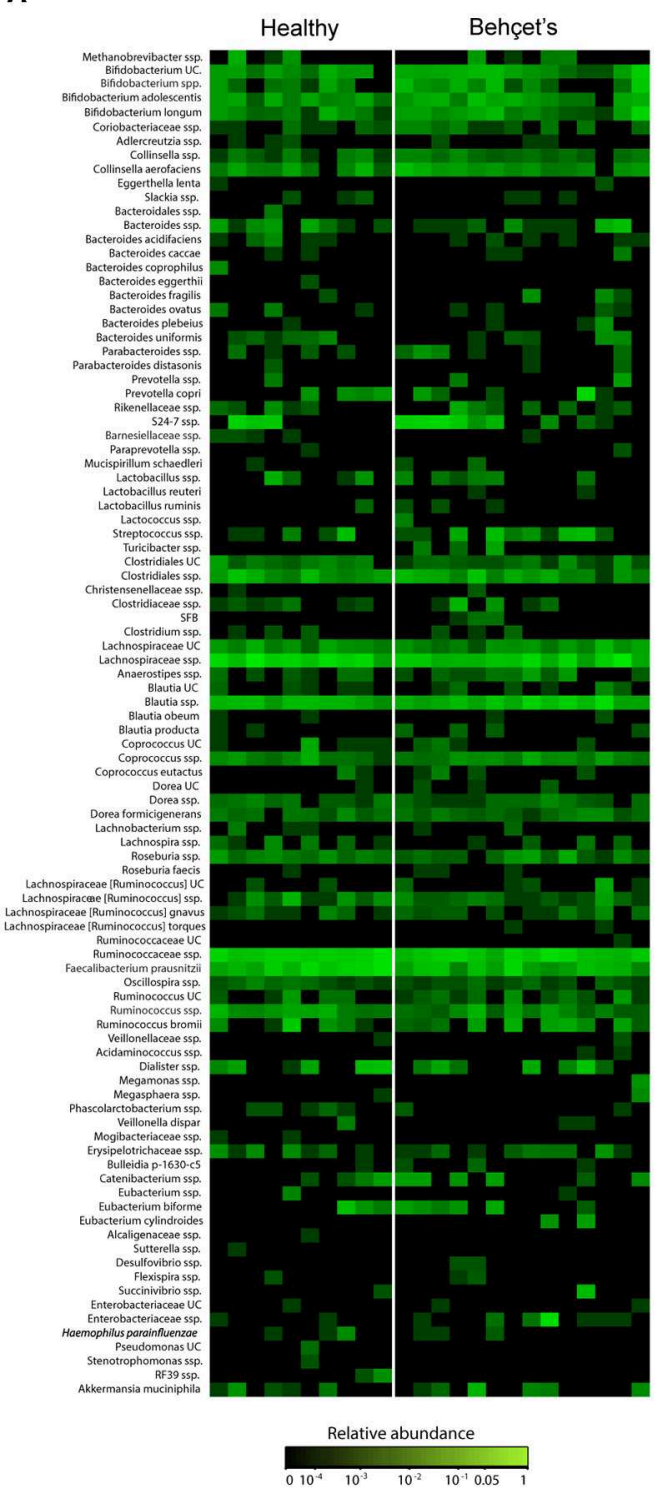

B

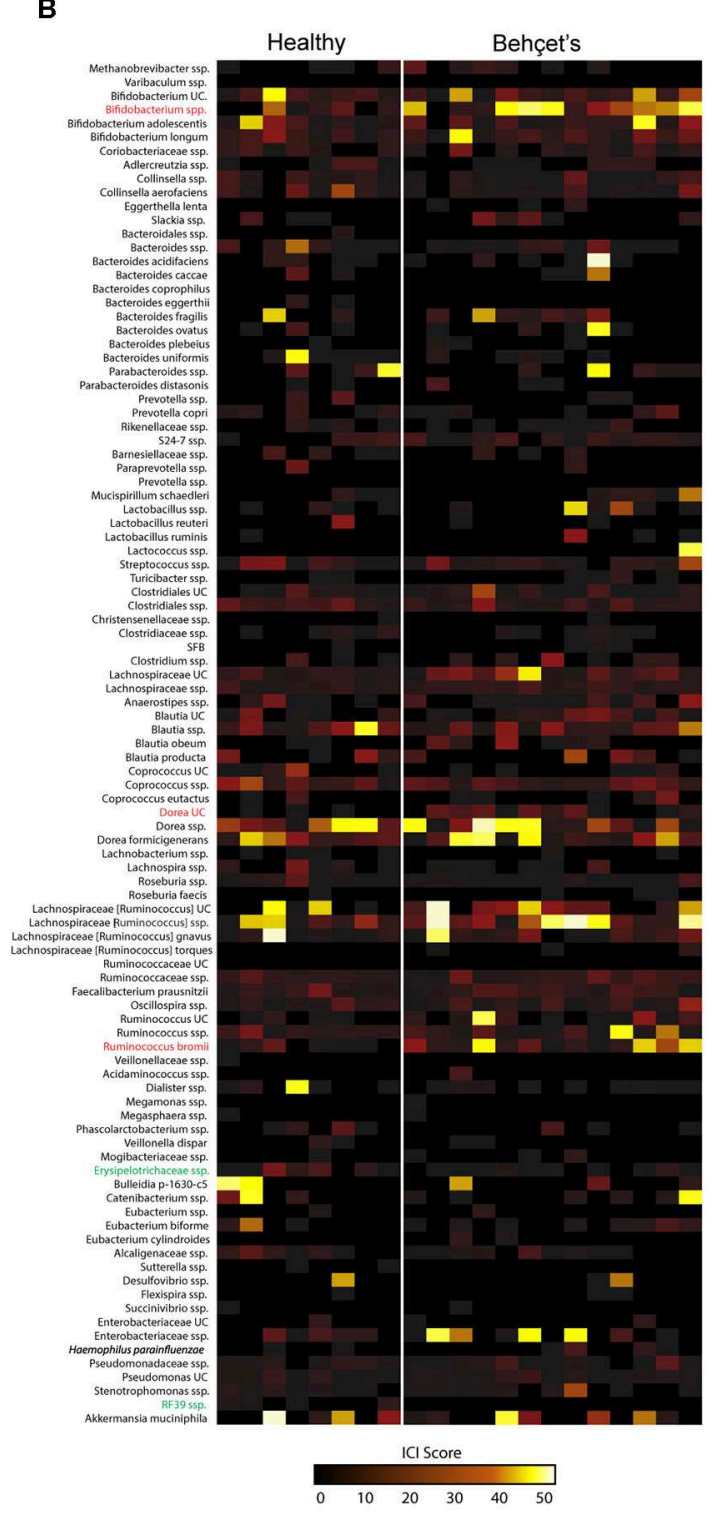

C

Behçet Healthy

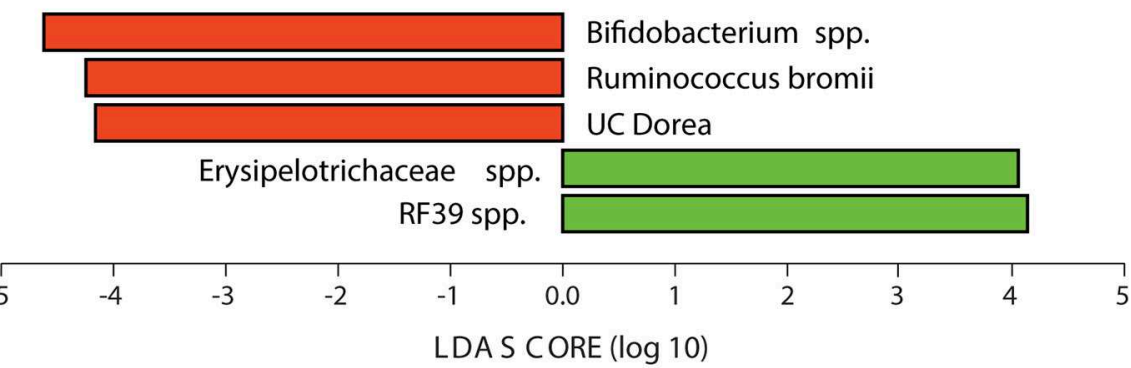

FIGURE 3 | (A) Abundance heatmap: heatmap depicting relative abundance of bacterial species in fecal bacteria from BD patients $(n=13$ samples) and HC $(n=8$ samples). The relative abundance heatmap is depicted on a logarithmic scale. UC, unclassified in the Greengenes reference database. (B) IgA-Coating Index (ICI) 
FIGURE 3 | heatmap: Heatmap depicting ICl scores of bacterial species in fecal bacteria from BD patients ( $n=13$ samples) and $\mathrm{HC}(n=8$ samples). Species that are significantly enriched in $\mathrm{BD}$ patients as compared to $\mathrm{HC}$ are depicted in red, species that are significantly IgA-coated in $\mathrm{HC}$ as compared to $\mathrm{BD}$ patients are depicted in green (LEfSe; $p<0.05)$. UC, unclassified in the Greengenes reference database. (C) Lefse plot: LEfSe comparisons of ICl scores of bacterial species from $\mathrm{BD}$ patients and HC. Taxa that are significantly enriched in BD patients are depicted in red, and taxa that are significantly enriched in the healthy controls are depicted in green. Significance levels for LEfSe were $p<0.05$ and Linear Discriminant Analysis (LDA) Score $>2$.

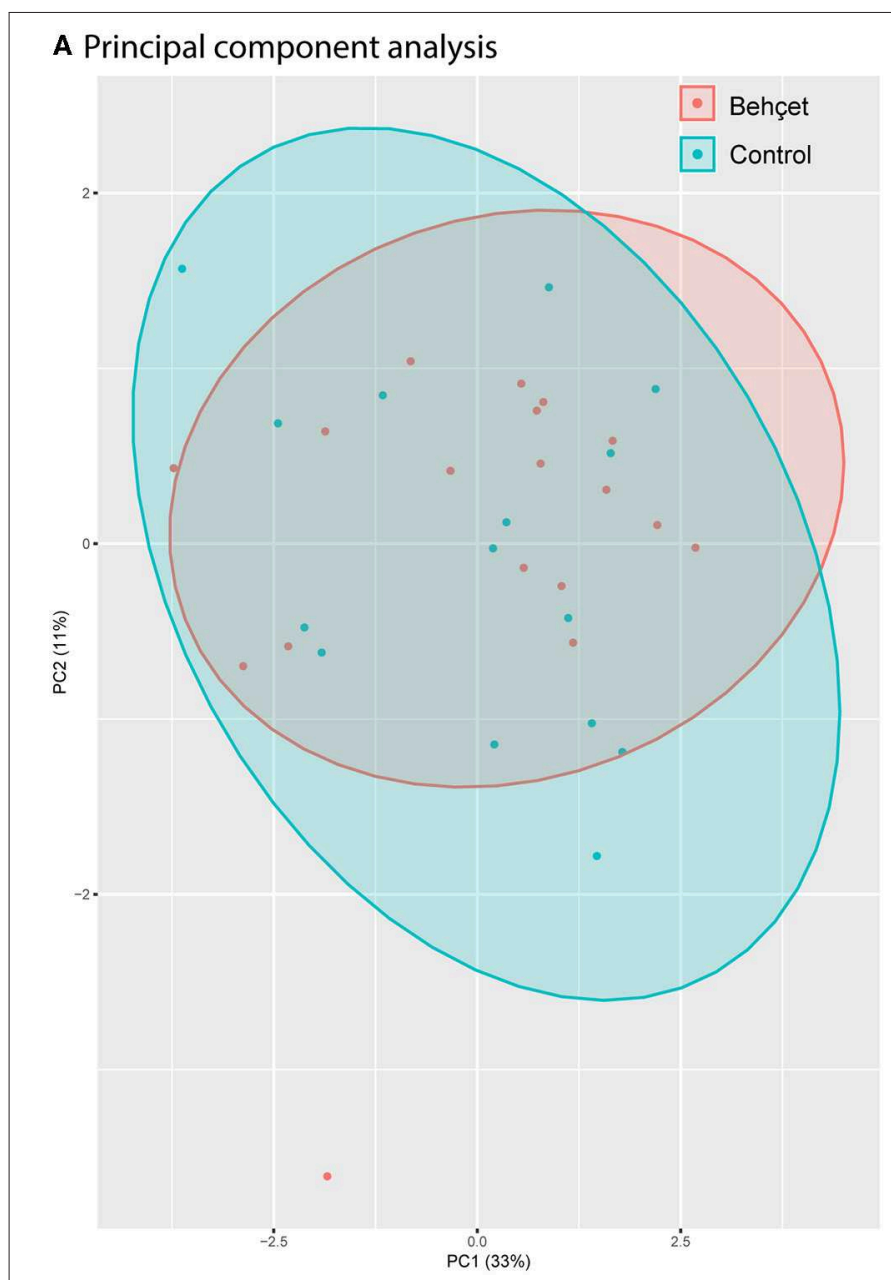

\section{B ANCOM analysis at family level}
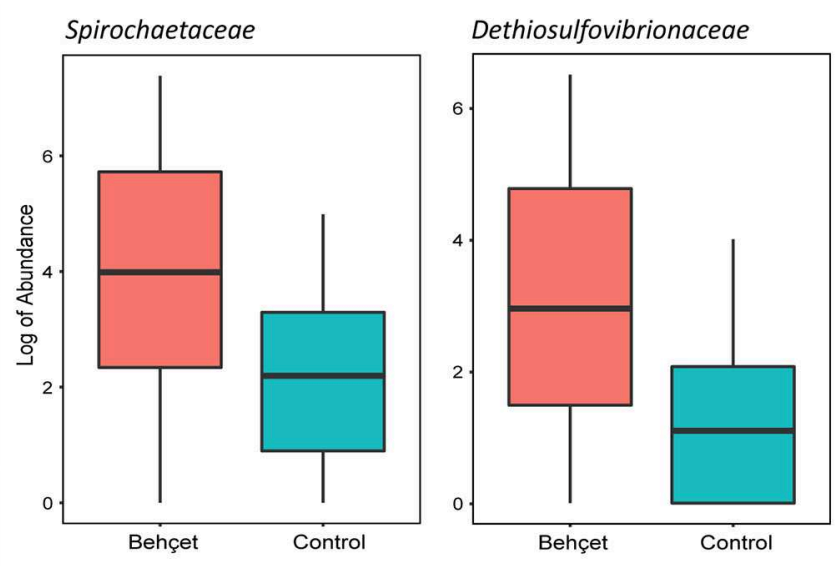

c ANCOM analysis at genus level
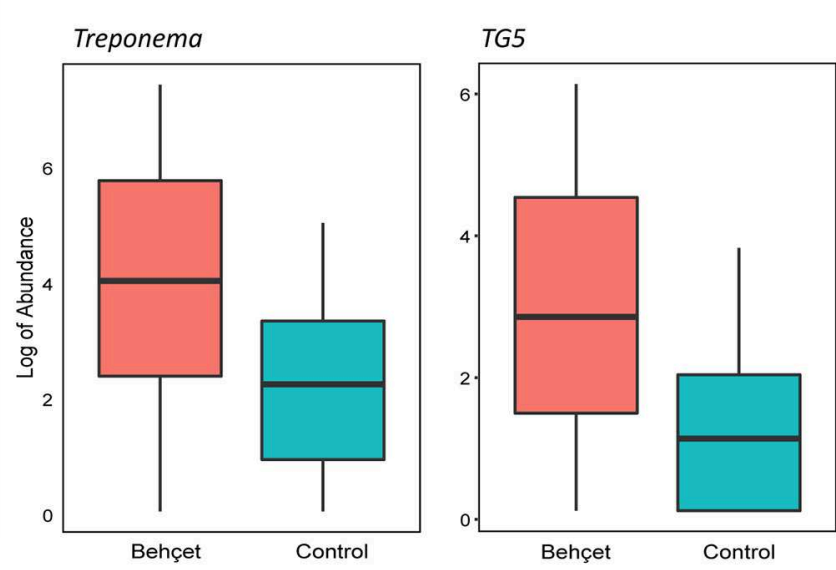

FIGURE 4 | (A) Principal components analysis (PCA), based on oral microbiota genera abundance, showing Dutch BD patients (orange) and Dutch HC (light blue). (B,C) Boxplots show the log of abundance of bacteria with significant increase in relative abundance (ANCOM analysis) in BD patients on family (B) and genus level (C). HC are represented with the light blue boxes; BD patients are shown as orange boxes.

a reduced fecal abundance of family Barnesiellaceae, and of unclassified Barnesiellaceae and Lachnospira genera. In separate cohort analysis, we demonstrate the Italian cohort drives the observed deviations. In the Dutch cohort the reduced fecal abundance of the family Barnesiellaceae and of unclassified Barnesiellaceae and Lachnospira genera does not reach statistical significance. Next to this, IgA-SEQ analysis revealed an association of $\mathrm{BD}$ with the presence of IgA-coated Bifidobacterium spp., Ruminococcus bromii and Dorea spp., and a reduced presence of IgA-coated Erysipelotrichaceae spp. and RF38 spp.

We demonstrated a decrease in abundance of Barnesiellaceae at both family and genus level and Lachnospira at genus level in patients with BD. Reduced abundance of Barnesiellaceae in stool is related with colitis in IL-22 deficient mice (30). Moreover, reduced abundance of Barnesiellaceae is inversely correlated with TNF- $\alpha$ levels serum in treated chronic HIV patients. (31) Barnesiellaceae might exert a protective antiinflammatory effect by reducing TNF- $\alpha$ level, one of the key and target cytokines in BD. Decreased abundance could, therefore, be a contributing factor to inflammation in BD. Decreased abundance of Lachnospira has not been associated with BD so far. Reduction of Lachnospira has been reported in CD with a decrease in production of short-chain fatty acid (SCFA), known to modulate inflammation through Tregs and suppression of the NF? $\beta$-pathway $(32,33)$. Interestingly, production of butyrate 
is significantly reduced in BD (10). This decreased production of butyrate may be mediated through reduced abundance of Lachnospira, thereby contributing to the inflammatory state in BD. In HLAB51 positive patients, decreased abundance of Barnesiellaceae and two unclassified families of the Clostridiales order, suggests that genetic background may influence the GM. This hypothesis is supported by healthy HLAB27+ siblings of spondyloarthritis patients, which display a distinct GM compared to healthy HLAB27- siblings (34).

In the Dutch cohort, IgA-SEQ analysis revealed increased IgA-coated Bifidobacterium, Dorea and Ruminococcus species, indicating that these microbes drive specific immunostimulatory responses and might be pathosymbionts in BD (14). Although Bifidobacterium has been found enriched in the fecal microbiomes of a Japanese BD cohort (11), a role as pathosymbiont for Bifidobacterium seems counterintuitive, since in other inflammatory disorders (such as CD) Bifidobacterium is believed to be a probiotic species that exerts immunomodulatory effects (35) Similar to Bifidobacterium, a pathogenic role for Ruminococcus bromii seems unlikely. This amylolytic bacteria is mainly known for its role in the degradation of starch, which is an essential component for the formation of SCFA, like propionate and butyrate. Both of these SCFAs induces IL10-producing Treg cells and, therefore, Ruminococcus bromii is generally considered as anti-inflammatory $(36,37)$. This is further supported by the decrease in relative abundance observed in CD patients $(38,39)$. The induced IgA-coating of both Bifidobacterium and Ruminococcus bromii in BD might, then, serve to enable efficient retention of the bacteria in the gut mucosa, contributing to sustainable homeostasis by dampening pro-inflammatory signaling in the host $(40,41)$. Nevertheless, a role as pathosymbiont in BD seems more likely for Dorea. Increased relative abundance of Dorea has been associated with an increment of intestinal permeability, thereby contributing to inflammation in inflammatory bowel diseases (42). The significance of increased IgA-coating of the respective bacteria needs to be further investigated, for instance, in mouse models.

Decreased diversity in microbiota has been found in CD (43), psoriatic arthritis (44) and it is associated with an inflammatory phenotype (45). A decreased diversity in microbiota has also been shown in BD patients (10). The Dutch cohort, in contrary, like the Japanese one (11), shows a comparable Shannon diversity between patients and HC. Analysis of the cohorts from two different countries showed no differences in both alpha- and beta- diversity in fecal microbiome between patients and HC. Differences among samples are evidently more strongly driven by country of origin instead of disease status. This could reflect either country-specific differences, such as diet or corticosteroid use in the Italian cohort, but also, differences in DNA extraction

\section{REFERENCES}

1. Sakane T, Takeno M, Suzuki N, Inaba G. Behçet's disease. N Engl J Med. (1999) 341:1284-91. doi: 10.1056/NEJM199910213411707

2. Nussenblatt RB. Uveitis in behçet's disease. Int Rev Immunol. (1997) 14:6779. doi: $10.3109 / 08830189709116845$ and PCR protocols. Moreover, "batch effect," due to sequencing Italian and Dutch individuals on different runs, cannot be excluded. Because of these differences, we decided to also perform analysis on the Dutch and Italian cohorts separately, which prevented to take advantage from increased statistical power on analysis on larger combined sample numbers.

Oral dysbiosis may lead to systemic inflammation, as in periodontitis (46). Despite similar alpha-and beta-diversity in the Dutch cohort, we distinguished increased abundance of families Spirochaetaceae and Dethiosulfovibrionaceae in oral microbiota of $\mathrm{BD}$ patients. Increased abundance of Dethiosulfovibrionaceae and Spirochaetaceae were both associated with periodontitis and endodontic infections, which are more prevalent in patients with severe BD (47-49).

\section{CONCLUSIONS}

In conclusion, we demonstrated decreased fecal abundance of Barnesiellaceae on family and genus level and of Lachnospira on genus level and increased oral abundance of Spirochaetaceae and Dethiosulfovibrionaceae on family level in patients with BD. The Italian cohort drives the observed deviations, which are only observed as a trend in the Dutch cohort. Next to this, increased fecal IgA-coating of Bifidobacterium and Ruminococcus bromii may reflect retention of anti-inflammatory species and increased fecal IgA-coating of Dorea may identify a pathosymbiont in BD. Additional studies, however, are warranted to relate intestinal microbes with the significance of ethnicity, diet, medication, and response with distinct pro- and inflammatory pathways in BD patients.

\section{DATA AVAILABILITY STATEMENT}

The datasets generated for this study can be found in the ENA database, study accession number PRJEB36332.

\section{ETHICS STATEMENT}

The studies involving human participants were reviewed and approved by Medical Ethical Committee of the Erasmus MC. The patients/participants provided their written informed consent to participate in this study.

\section{AUTHOR CONTRIBUTIONS}

TH, JL, JK, PH, and HL designed the experiment. MZ and GM performed the IgA-SEQ analysis. R-MB, AF, JG, and $\mathrm{CH}$ analyzed the data (both cohorts). CC, MS, CP, MD, and GE are responsible for the Italian experiment and collecting of data. All authors read and approved the final manuscript.

3. Evereklioglu C. Current concepts in the etiology and treatment of behçet disease. Surv Ophthalmol. (2005) 50:297350. doi: 10.1016/j.survophthal.2005.04.009

4. Gül A. Behçet's disease as an autoinflammatory disorder. Curr Drug Targets Inflamm Allergy. (2005) 4:81-3. doi: 10.2174/15680100536 22894 
5. Mumcu G, Inanc N, Aydin SZ, Ergun T, Direskeneli H. Association of salivary S. Mutans colonisation and mannose-binding lectin deficiency with gender in behçet's disease. Clin Exp Rheumatol. (2009) 27(2 Suppl. 53):S32-6.

6. Mochizuki M, Suzuki N, Takeno M, Nagafuchi H, Harada T, Kaneoka H, et al. Fine antigen specificity of human gamma delta $\mathrm{T}$ cell lines (V gamma 9+) established by repetitive stimulation with a serotype (KTH-1) of a grampositive bacterium, Streptococcus sanguis. Eur J Immunol. (1994) 24:153643. doi: $10.1002 /$ eji.1830240712

7. Hirohata S, Oka H, Mizushima Y. Streptococcal-related antigens stimulate production of IL6 and interferon-gamma by T cells from patients with behcet's disease. Cell Immunol. (1992) 140:410-9. doi: 10.1016/0008-8749(92)90207-6

8. Skin hypersensitivity to streptococcal antigens and the induction of systemic symptoms by the antigens in behçet's disease-a multicenter study. The behcet's disease research committee of Japan. J Rheumatol. (1989) 16:506-11.

9. Calgüneri M, Kiraz S, Ertenli I, Benekli M, Karaarslan Y, Celik I. The effect of prophylactic penicillin treatment on the course of arthritis episodes in patients with behçet's disease. A randomized clinical trial. Arthritis Rheum. (1996) 39:2062-5. doi: 10.1002/art.1780391216

10. Consolandi C, Turroni S, Emmi G, Severgnini M, Fiori J, Peano C, et al. Behçet's syndrome patients exhibit specific microbiome signature. Autoimmun Rev. (2015) 14:269-76. doi: 10.1016/j.autrev.2014.11.009

11. Shimizu J, Kubota T, Takada E, Takai K, Fujiwara N, Arimitsu N, et al. Bifidobacteria abundance-featured gut microbiota compositional change in patients with behcet's disease. PLoS ONE. (2016) 11:e0153746. doi: 10.1371/journal.pone.0153746

12. Seoudi N, Bergmeier LA, Drobniewski F, Paster B, Fortune F. The oral mucosal and salivary microbial community of behçet's syndrome and recurrent aphthous stomatitis. J Oral Microbiol. (2015) 7:27150. doi: 10.3402/jom.v7.27150

13. Palm NW, de Zoete MR, Cullen TW, Barry NA, Stefanowski J, Hao L, et al. Immunoglobulin A coating identifies colitogenic bacteria in inflammatory bowel disease. Cell. (2014) 158:1000-10. doi: 10.1016/j.cell.2014.08.006

14. Viladomiu M, Kivolowitz C, Abdulhamid A, Dogan B, Victorio D, Castellanos JG, et al. IgA-coated E. coli enriched in crohn's disease spondyloarthritis promote TH17-dependent inflammation. Sci Transl Med. (2017) 9:eaaf9655. doi: 10.1126/scitranslmed.aaf 9655

15. Criteria for diagnosis of behçet's disease. International study group for behçet's disease. Lancet. (1990) 335:1078-80. doi: 10.1016/0140-6736(90)92643-V

16. Buelow E, Gonzalez TB, Versluis D, Oostdijk EAN, Ogilvie LA, van Mourik MSM, et al. Effects of selective digestive decontamination (SDD) on the gut resistome. J Antimicrob Chemother. (2014) 69:2215-23. doi: 10.1093/jac/dku092

17. Bogaert D, Keijser B, Huse S, Rossen J, Veenhoven R, van Gils E, et al. Variability and diversity of nasopharyngeal microbiota in children: a metagenomic analysis. PLoS ONE. (2011) 6:e17035. doi: 10.1371/journal.pone.0017035

18. Paganelli FL, Luyer M, Hazelbag CM, Uh H-W, Rogers MRC, Adriaans $\mathrm{D}$, et al. Roux-Y gastric bypass and sleeve gastrectomy directly change gut microbiota composition independent of operation type. Sci. Rep. (2018) 9:10979. doi: $10.1101 / 395657$

19. Fadrosh DW, Ma B, Gajer P, Sengamalay N, Ott S, Brotman RM, et al. An improved dual-indexing approach for multiplexed $16 \mathrm{~S}$ rRNA gene sequencing on the illumina MiSeq platform. Microbiome. (2014) 2:6. doi: 10.1186/2049-2618-2-6

20. Magoč T, Salzberg SL. FLASH: fast length adjustment of short reads to improve genome assemblies. Bioinformatics. (2011) 27:2957-63. doi: 10.1093/bioinformatics/btr507

21. Caporaso JG, Kuczynski J, Stombaugh J, Bittinger K, Bushman FD, Costello EK, et al. QIIME allows analysis of high-throughput community sequencing data. Nat Methods. (2010) 7:335-6. doi: 10.1038/nmeth.f.303

22. Edgar RC. Search and clustering orders of magnitude faster than BLAST. Bioinformatics. (2010) 26:2460-1. doi: 10.1093/bioinformatics/ btq461

23. Caporaso JG, Lauber CL, Walters WA, Berg-Lyons D, Huntley J, Fierer $\mathrm{N}$, et al. Ultra-high-throughput microbial community analysis on the illumina HiSeq and MiSeq platforms. ISME J. (2012) 6:16214. doi: $10.1038 /$ ismej.2012.8

24. R Core Team. R: The R Project for Statistical Computing. Available online at: https://www.r-project.org/ (accessed January 13, 2019).
25. Gloor GB, Reid G. Compositional analysis: a valid approach to analyze microbiome high-throughput sequencing data. Can J Microbiol. (2016) 62:692-703. doi: 10.1139/cjm-2015-0821

26. Wickham H. ggplot2. New York, NY: Springer (2009). doi: 10.1007/978-0-387-98141-3

27. Segata N, Huttenhower C. Toward an efficient method of identifying core genes for evolutionary and functional microbial phylogenies. PLOS ONE. (2011) 6:e24704. doi: 10.1371/journal.pone.0024704

28. Mandal S, Van Treuren W, White RA, Eggesbø M, Knight R, Peddada SD. Analysis of composition of microbiomes: a novel method for studying microbial composition. Microb Ecol Health Dis. (2015) 26:27663. doi: 10.3402/mehd.v26.27663

29. Storey JD, Tibshirani R. Statistical significance for genomewide studies. Proc Natl Acad Sci USA. (2003) 100:9440-5. doi: 10.1073/pnas.1530509100

30. Zenewicz LA, Yin X, Wang G, Elinav E, Hao L, Zhao L, et al. IL-22 deficiency alters colonic microbiota to be transmissible and colitogenic. J Immunol. (2013) 190:5306-12. doi: 10.4049/jimmunol.1300016

31. Dinh DM, Volpe GE, Duffalo C, Bhalchandra S, Tai AK, Kane A $\mathrm{V}$, et al. Intestinal microbiota, microbial translocation, and systemic inflammation in chronic HIV infection. J Infect Dis. (2015) 211:1927. doi: 10.1093/infdis/jiu409

32. Wang Y, Gao X, Ghozlane A, Hu H, Li X, Xiao Y, et al. Characteristics of faecal microbiota in paediatric crohn's disease and their dynamic changes during infliximab therapy. J Crohns Colitis. (2018) 12:33746. doi: 10.1093/ecco-jcc/jjx153

33. Richards JL, Yap YA, McLeod KH, Mackay CR, Mariño E. Dietary metabolites and the gut microbiota: an alternative approach to control inflammatory and autoimmune diseases. Clin Transl Immunol. (2016) 5:e82. doi: 10.1038/cti.2016.29

34. Breban M, Tap J, Leboime A, Said-Nahal R, Langella P, Chiocchia G, et al. Faecal microbiota study reveals specific dysbiosis in spondyloarthritis. Ann Rheum Dis. (2017) 76:1614-22. doi: 10.1136/annrheumdis-2016-211064

35. Saez-Lara MJ, Gomez-Llorente C, Plaza-Diaz J, Gil A. The role of probiotic lactic acid bacteria and bifidobacteria in the prevention and treatment of inflammatory bowel disease and other related diseases: a systematic review of randomized human clinical trials. Biomed Res Int. (2015) 2015:505878. doi: 10.1155/2015/505878

36. Ze X, Duncan SH, Louis P, Flint HJ. Ruminococcus bromii is a keystone species for the degradation of resistant starch in the human colon. ISME J. (2012) 6:1535-43. doi: 10.1038/ismej.2012.4

37. Arpaia N, Campbell C, Fan X, Dikiy S, van der Veeken J, deRoos P, et al. Metabolites produced by commensal bacteria promote peripheral regulatory T-cell generation. Nature. (2013) 504:451-5. doi: 10.1038/nature12726

38. Mondot S, Kang S, Furet JP, Aguirre de Carcer D, McSweeney C, Morrison M, et al. Highlighting new phylogenetic specificities of crohn's disease microbiota. Inflamm Bowel Dis. (2011) 17:185-92. doi: 10.1002/ibd.21436

39. Kang S, Denman SE, Morrison M, Yu Z, Dore J, Leclerc M, et al. Dysbiosis of fecal microbiota in crohn's disease patients as revealed by a custom phylogenetic microarray. Inflamm Bowel Dis. (2010) 16:203442. doi: 10.1002/ibd.21319

40. Brandtzaeg P. Secretory IgA: designed for anti-microbial defense. Front Immunol. (2013) 4:222. doi: 10.3389/fimmu.2013.00222

41. Donaldson GP, Ladinsky MS, Yu KB, Sanders JG, Yoo BB, Chou W-C, et al. Gut microbiota utilize immunoglobulin A for mucosal colonization. Science. (2018) 360:795-800. doi: 10.1126/science.aaq0926

42. Maharshak N, Ringel Y, Katibian D, Lundqvist A, Sartor RB, Carroll IM, et al. Fecal and mucosa-associated intestinal microbiota in patients with diarrhea-predominant irritable bowel syndrome. Dig Dis Sci. (2018) 63:18909. doi: 10.1007/s10620-018-5086-4

43. Manichanh C, Rigottier-Gois L, Bonnaud E, Gloux K, Pelletier E, Frangeul L, et al. Reduced diversity of faecal microbiota in crohn's disease revealed by a metagenomic approach. Gut. (2006) 55:205-11. doi: 10.1136/gut.2005.073817

44. Scher JU, Ubeda C, Artacho A, Attur M, Isaac S, Reddy SM, et al. Decreased bacterial diversity characterizes the altered gut microbiota in patients with psoriatic arthritis, resembling dysbiosis in inflammatory bowel disease. Arthritis Rheumatol. (2015) 67:128-39. doi: 10.1002/art.38892

45. Le Chatelier E, Nielsen T, Qin J, Prifti E, Hildebrand F, Falony G, et al. Richness of human gut microbiome correlates with metabolic markers. Nature. (2013) 500:541-6. doi: 10.1038/nature12506 
46. Arimatsu K, Yamada H, Miyazawa H, Minagawa T, Nakajima M, Ryder $\mathrm{MI}$, et al. Oral pathobiont induces systemic inflammation and metabolic changes associated with alteration of gut microbiota. Sci Rep. (2014) 4:4828. doi: 10.1038/srep04828

47. Vartoukian SR, Palmer RM, Wade WG. The division "Synergistes". Anaerobe. (2007) 13:99-106. doi: 10.1016/j.anaerobe.2007.05.004

48. Dashper SG, Seers CA, Tan KH, Reynolds EC. Virulence factors of the oral spirochete Treponema denticola. J Dent Res. (2011) 90:691703. doi: $10.1177 / 0022034510385242$

49. Habibagahi Z, Khorshidi H, Hekmati S. Periodontal health status among patients with behçet's disease. Scientifica. (2016) 2016:7506041. doi: 10.1155/2016/7506041
Conflict of Interest: The authors declare that the research was conducted in the absence of any commercial or financial relationships that could be construed as a potential conflict of interest.

Copyright (c) 2020 van der Houwen, van Laar, Kappen, van Hagen, de Zoete, van Muijlwijk, Berbers, Fluit, Rogers, Groot, Hazelbag, Consolandi, Severgnini, Peano, D'Elios, Emmi and Leavis. This is an open-access article distributed under the terms of the Creative Commons Attribution License (CC BY). The use, distribution or reproduction in other forums is permitted, provided the original author(s) and the copyright owner(s) are credited and that the original publication in this journal is cited, in accordance with accepted academic practice. No use, distribution or reproduction is permitted which does not comply with these terms. 